\title{
MODELOS ALGEBRAICOS DE WALLIS PARA COMPONER SÓLIDOS DE DIMENSIÓN INFINITA Y VOLUMEN DETERMINADO
}

Piedad YUSTE

Resumen: Inspirado en la construcción del sólido hiperbblico de Torricelli, John Wallis intentó obtener resultados semejantes utilizando sus series algebraicas infinitas. Estas se componian de sumas de lineas y de planos de espesor infinitésimo, pero cuya magnitud se ordenaba, bien en función de los términos de una progresión geométrica decreciente o según sucesiones de números positivos, racionales $e$ irracionales. En este trabajo se muestran ambos desarrollos.

Descriptores: Solido hiperbólico, John Wallis, sólidos infinitos.

Abstract: Inspired b y the Torricelli construction of Hyperbolic Solid, John Wallis tried to obtain similar results using their infinite algebraic series. These consisted on the sums of segments and surfaces but with an infinitesimal thickness; its magnitude was ordered in function to the terms of a geometric progression or according to the successions of positive, rational and irrational numbers. In this paper these developments are shown.

Key words: Hyperbolic Solid, algebraic, John Wallis, infinite solids.

La construcción del sólido hiperbólico de Torricelli maravilló a John Wallis, tanto, que dedicó muchas horas de estudio a investigar las propiedades necesarias que habrían de tener las superficies y los cuerpos sometidos a las condiciones establecidas por el sabio de Faenza. Y para conseguirlo, usó los mismos indivisibles con cantidad imaginados por éste, ordenándolos y distribuyéndolos de dos formas diferentes, acordes también a esas dos nociones de infinito que se habían insinuado en las deducciones de Torricelli: una, al diseñar el sólido hiperbólico y otra, cuando expresó la cuadratura de la parábola 
en función de los términos de una progresión geométrica decreciente. Los modelos que Wallis propuso, separados en el tiempo, poseen algo en común: el lenguaje algebraico de sus algoritmos.

\section{El infinito actualizado en el sólido hiperbólico de Torricell}

En 1641 y con el método de los indivisibles ${ }^{1}$, Torricelli descubrió un teorema notabilísimo que expuso enseguida a Cavalieri. En él consiguió probar que un cuerpo sólido de altura infinita posee, sin embargo, un volumen fijo y determinable. Tan extraña y paradójica construcción cautivó inmediatamente a su maestro ${ }^{2}$. Sin embargo, aunque este descubrimiento fue conocido dos años más tarde por los matemáticos del círculo de Mersenne, su exposición y formalización no se publicó hasta 1919, en las Opere (vol. I, parte 1, pp. 191221) ${ }^{3}$. Torricelli comienza este trabajo comentando que

«... existe un sólido de longitud infinita pero dotado de una sutileza tal que, aun prolongado al infinito, no supera la cantidad de un pequeño cilindron ${ }^{4}$.

Se trata de un cuerpo engendrado por la rotación de uno de los brazos de la hipérbola equilátera alrededor de su asíntota vertical, tomada como eje, y al cual se añade, a modo de peana, un cilindro que descansa en la otra asíntota.

1 Método ideado por Bonaventura Cavalieri para hallar cuadraturas y cubaturas; consiste en establecer proporciones entre figuras (planas o sólidas) y sus respectivas colecciones de indivisibles (líneas y planos). Desarrolló esta técnica en la Geometria indivisibilibus continuorum nova quadam ratione promota (Bologna, 1635) y en Exercitationes Geometricae Sex (Bologna, 1647). De la primera existe una versión en italiano: Geometria degli Indivisibili, con introducción y notas a cargo de Lombardo-Radice, UTET, 1966. De la segunda, tenemos la edición preparada por E. Giusti, con su valiosa introducción: "Bonaventura Cavalieri and the Theory of Indivisibles", Introductory Essay to Reprint of Exercitationes Geometricae Sex, UMI, Roma, Cremonese, 1980.

2 Ver P. Mancosu y E. Vailati, "Torricelli's Infinitely Long Solid and its Philosophical Reception in the Seventeenth Century", Isis (1991) vol. 82, pp. 50-70. Mancosu, Philosophy of Mathematics ef Ma thematical Practice in the Seventeenth Century, Oxford Univ. Press, 1996.

3 Aclaración indicada por un especialista. Edición de las Opere a cargo de G. Loria y G. Vassura en 4 vols., Faenza. La Opera Geometrica (Firenze, 1644) no contenía este trabajo. Sí está contenido en Opere Scelte, edición a cargo de Lanfranco Belloni, UTET,1975, pp. 421-475.

4 Opere Scelte, p. 422. 


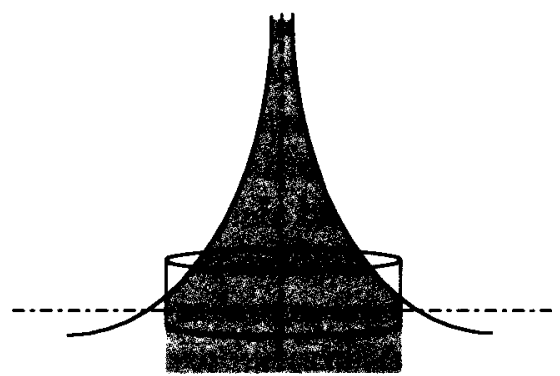

Figura 1

La explicación de Torricelli se apoya en la noción de los indivisibles curvos, ya que así es como llama a las periferias de los círculos (o circunferencias), si trabajamos en el plano, y a las periferias de los cilindros, de las esferas y de los conos, cuando manejamos sólidos. Reclama para estos indivisibles curvos algo que Cavalieri nunca se había atrevido a mencionar: un "espesor siempre igual y uniforme» ${ }^{5}$. Pero no garantizó su existencia con algún axioma o postulado previo; únicamente se limitó a definirlos, o más bien a nombrarlos, pues su exposición no va más allá de una sencilla aprehensión intuitiva. Tomó los agregados de esos indivisibles y supuso que al tener cada uno de ellos cierta magnitud o grosor ${ }^{6}$, juntos lograrían componer el área de un círculo y el volumen de un sólido, según las periferias fueran sólo líneas o únicamente superficies. Torricelli lograba de este modo superar la repugnancia de Cavalieri a identificar conjuntos de líneas y de planos con sus respectivas figuras ${ }^{7} \mathrm{y}$ restauraba la ansiada homogeneidad entre el todo y sus partes.

5 Para Cavalieri, los puntos, las líneas y los planos no poseen magnitud o anchura; son como los define Euclides en los Elementos I (Exercitatio III, p. 180). Esto le impediría tratar los agregados de indivisibles como sumas. Giusti (1980, p. 28).

6 El que Torricelli dotara a los indivisibles de cierto espesor o magnitud se debió a las contradicciones detectadas en el uso del método de indivisibles y que expuso en De indivisibilium doctrina perperam usurpata, obra no publicada en vida de Torricelli y la cual se encuentra en Opere Scelte, pp. 494 y ss. Ver también F. De Gandt (1984): «Naissance et métamorphoses d'une théorie mathématique: la géometrie des indivisibles en Italie (Galilée, Cavalieri, Torricelli)", Sciences et Techniques en perspective, Univ. de Nantes, vol. IX, pp. 179-226. De Gandt (1987): L'oeuvre de Torricelli: Science galiléenne et nouvelle géometrie, Fac. des Lettres et Sciences Humaines de Nice, num. 32, serie 1.

7 En la Geometria indivisibilibus II, III, Cavalieri destacó únicamente que: "Las figuras planas tienen entre sí la misma relación que guardan todas sus líneas, tomadas con una referencia cualquiera; y las figuras sólidas poseen la misma razón que tienen todos sus planos, tomados respecto a uno cualquiera de ellos", evitando a toda costa que alguien pudiera acusarle de identificar las figuras con los agregados de sus indivisibles. 
La medida del volumen de un cuerpo se determina entonces por el conjunto de todos los planos superpuestos que lo componen y también por el agregado de formas análogas concéntricas situadas en su interior. Así, un cilindro está constituido, bien por una cantidad indefinida de círculos apilados uno sobre otro ${ }^{8}$; bien por una multitud de cilindros huecos, cuyos diámetros aumentan progresivamente. $Y$ en ambos casos, el cardinal de ese agregado de indivisibles podría ser el infinito que actualiza todos sus elementos, aquí y abora. El mismo infinito con el que Cavalieri - muy a su pesar- habría caracterizado a las magnitudes omnes ${ }^{9}$.

Una vez definido ese cuerpo tan singular al que denominaba solido acuto hyperbolico, Torricelli decidió medir su volumen del mismo modo que había hecho en otras ocasiones ${ }^{10}$ : estableciendo razones entre los respectivos agregados de indivisibles que componen un cilindro, un cono o una esfera. Su razonamiento se asienta sobre 5 lemas demostrados al modo de los teoremas y derivados del estudio de las propiedades de una hipérbola equilátera. Al final concluye que el conjunto de todos los cilindros que conforman el sólido equivale al agregado de los círculos que componen un cilindro de dimensiones definidas ${ }^{11}$.

8 Heath (The Thirteen Books, Dover, N.Y., 1956, p. 368) muestra el texto recogido por Plutarco (De Comm. Not. adv. Stoicos XXXIX, 3) en el que Demócrito afirma que el cono parece estar hecho de secciones iguales. Ver la edición de los Elementos a cargo de M. ${ }^{2} \mathrm{~L}$. Puertas y L. Vega, Gredos, Madrid, 1991-1996.

9 Cavalieri denominó omnes a los agregados de líneas y de planos; primero intentó demostrar en las proposiciones I y III de la Geometria II que consistían en un nuevo tipo de magnitudes, cumpliendo entonces los requisitos fijados por Eudoxo en las definiciones 4 y 5 del libro $V$ de los Elementos. Aunque el número de elementos que componía cada uno de esos agregados pareciera ser infinito, estarían, sin embargo, limitados por el contorno de las figuras; esto los haría ser relativamente infinitos o indefinidos. En realidad, Cavalieri temía introducir infinitos en geometría y contravenir con ello, tanto el dictum de Aristóteles («no hay razón ni proporción entre lo finito y lo infinito", Del Cielo I, 6, 273b-274 a) como las tesis desarrolladas en los Elementos. Cf. Exercitatio Tertia, cap. VIII, p. 201, donde Cavalieri expone las objeciones presentadas por Güldin en Centrobaryca (también llamada De centro gravitatis) a su método de indivisibles. Existe abundante bibliografia al respecto: K. Andersen, "Cavalieri's Method of Indivisibles", Archive for the History of Exact Sciences (1984), Feb., pp. 291-367; F. De Gandt, "Cavalieri's Indivisibles and Euclid's Canons", en P. Barker, R. Ariew eds., Revolution and Continuity. Essays in the History and Philosophy of Modern Science, The Catolic Univ. of America Press, Washington, 1991, pp. 157-192. A. Malet, From Indivisibles to Infinitesimals. Studies on the Seventeenth-Century Mathematizations of Infinitely Small Quantities, Univ. Autonoma de Barcelona, Barcelona, 1996

10 Opere Scelte, pp. $421-475$
Ibid., p. 444. 
Aparece aquí la imagen intuitiva de un cuerpo geométrico en cuyo interior hallamos una multitud ordenada de cilindros; estos aumentan en altura al tiempo que disminuyen sus bases respectivas, desde el más externo, hasta el que, prolongado infinitamente hacia arriba, alcanza el eje y se confunde con él ${ }^{12}$. Sin embargo, no sabemos cuántos cilindros componen el sólido ni cuántos círculos hay en el cilindro. Torricelli, usando el lenguaje de Cavalieri, afirma que son todos, pero pronto advertimos que en ningún momento indica que sean infinitos en número, pues esto hubiera añadido otra dificultad: la de efectuar cálculos de proporciones comparando cantidades finitas e infinitas. Por tanto, la construcción del sólido hiperbólico no introduce ningún lema que justifique el uso de sumas de indivisibles (quizá Torricelli no dio con él) ni tampoco alude a que la cantidad de estos sea infinita. En todo caso, los agregados de indivisibles se encuentran detenidos por los bordes de las figuras y esto los hace diferentes a los conjuntos numerables de Cantor ${ }^{13}$.

El sólido de Torricelli, infinitamente largo en el sentido que niega Aristóteles ${ }^{14}$, se ubica en un espacio también infinito. Así, la actualización de todos los cilindros contenidos en su interior supone su correlato espacial, es decir, la existencia de un espacio ilimitado Este asunto fue el origen de nuevas polémicas y discusiones entre los matemáticos más prestigiosos del siglo XVII, algunos de los cuales exigían que las proposiciones geométricas se fundamentasen en un espacio físico real ${ }^{15}$ al cual consideraban de tamaño limitado.

12 Torricelli comenta (Opere Scelte, p. 506): «Lo que dice Galileo respecto a un punto igual a una línea es verdad en nuestro sólido hiperbólico, pues es cierto que el eje infinitamente largo es igual a una superficie cilíndrica, o bien, a un círculo." (Torricelli alude aqui a la paradoja de la escudilla, concebida por Galileo para objetar algunos de los aspectos del método de indivisibles; cf. Discorsi, Opere VIII, pp. 73-76)

13 Lombardo-Radice, en la introducción a la Geometria degli indivisibili (Utet, pp. 25-26), destaca que Cavalieri imaginó sus agregados de indivisibles como conjuntos de infinitos elementos agrupados según una propiedad característica, anticipando a Cantor, y subraya que fue el primer pensador y también el primer matemático que distinguió un conjunto de infinitos elementos de su suma. G. Baroncelli es de la misma opinión (Bonaventura Cavalieri: Carteggio. Olschki, Firenze, 1987, pp. 22-23). Giusti (1980, p. 27) se muestra contrario a esta interpretación.

14 El único infinito que concibe Aristóteles es el potencial: "lo continuo se divide al infinito, pero hacia lo mayor no hay infinito" (Fisica IV 6, 207 b); asimismo, ues evidente también que no es posible que el infinito exista como algo dotado de existencia actual.» (Física III 5,204 a)

15 Ver Mancosu (1996, pp. 129-145; aquí, p. 139) 


\section{El infinito potencial en la cuadratura de la parábola}

En De dimensione parabolae ${ }^{16}$ Torricelli examina la superficie del segmento parabólico de diversas maneras: prueba primero los resultados de Arquímedes ${ }^{17}$ por el método clásico de exhausción y después, apoyándose en el uso de indivisibles, consigue demostrar de otras siete formas diferentes, esas mismas consecuencias arquimedianas. El Lema XVIII ${ }^{18}$ le va a permitir sustituir el principio de inducción ${ }^{19}$ que enunciara en su día Cavalieri, por sumas de un número indeterminado de indivisibles. $Y$ en lugar de afirmar que las. sumas de esos elementos están en la misma razón que sus figuras respectivas, sostendrá que el conjunto de esas líneas o de esos planos, o bien su suma, no es otra cosa que la figura misma, ya sea plana o sólida.

Uno de los procedimientos para hallar la cuadratura de la parábola se basa en el concepto de progresión geométrica decreciente: Torricelli aproxima a la curva sucesivos triángulos de magnitud cada vez menor, ordenados según una proporción numérica; su suma nos facilita la medida del segmento parabólico. Este argumento recuerda la construcción geométrica que, según parece, debió concebir Hipócrates de Khíos para demostrar que los círculos son como los

\section{Cf. Opere Scelte.}

17 La cuadratura de la parábola, cf. Dijksterhuis: Archimedes, Princeton Univ. Press, Princeton, New Jersey, 1987.

18 «Si una primera magnitud está a una segunda como una tercera está a una cuarta, esto cuantas veces se quiera; y siendo todas las primeras y también todas las terceras, iguales entre sí [respectivamente]. Decimos que todas las primeras juntas, están a todas las segundas, como todas las terceras están a todas las cuartas." Arquímedes dispuso de otro muy similar enunciado en Sobre Conoides y esferoides, proposición 1, y en El Método; ambos son variaciones del ya mencionado Elementos V.12. Cf. El Método, L. Vega y M. L. Puertas (eds.), Alianza, 1986, p. 39.

19 Denominado por Andersen principio ut-unum (op. cit., p. 317); fue definido por Cavalieri en forma de corolario tras la exposición del teorema IV del libro II: "...como uno de los antecedentes está a uno de los consecuentes, así todos los antecedentes están a todos los consecuentes.» (El método inductivo es aquí incompleto) Este principio se expresa de modo prácticamente idéntico al teorema V.12 de Euclides, lo cual ha provocado que numerosos comentaristas, como Baroncelli (1987, pp. 22-23), interpreten los agregados de indivisibles de Cavalieri en función de sumas. Rechazan esta explicación Andersen (1984), Lombardo-Radice (1966, p.24) y Giusti (1980, p. 28)

20 Cf. L. Vega, La trama de la demostración, Alianza, Madrid, 1990. También, Knorr, "Infinity and Continuity: The Interaction of Mathematics and Philosophy in Antiquity", Kretzmann, N. (ed.): Infinity and Continuity in Ancient and Medieval Thought, Ithaca, New York, 1982, pp. 112-145. Kouremenos, "Mathematical Rigor and the Origin of the Exhaustion Method", Centaurus 39 (1997), pp. 230-252 
cuadrados de sus diámetros ${ }^{20}$ y que se basa, a su vez, en una primer esbozo de lo que después se dio en llamar principio de bisección ${ }^{21}$. Asimismo, Torricelli tomará como referencia la construcción realizada por Arquímedes en La cuadratura de la parábola ${ }^{22}$.

Comienza la exposición enunciando unos lemas referentes a progresiones, antes de probar, en la proposición XV, que el segmento parabólico mide 4/3 del triángulo inscrito. Los lemas XXIV y XXV muestran el modo de acoplar los términos de una progresión infinita decreciente entre dos líneas concurrentes, mientras de los otros se desprende que existe una línea finita igual a la suma de todos ellos y que la primera magnitud es media proporcional entre la primera diferencia y el agregado de todas esas magnitudes ${ }^{23}$.

Es más, en el Escolio al lema XXVII añade: «Sea cualquier número de magnitudes, finito o infinito, si cada una es mayor que la que le sucede, decimos entonces que la primera de ellas es igual al agregado de todas las diferencias junto a la última.» Ésta - dice-o no existirá o será un punto. Es decir, cualquier progresión geométrica decreciente contiene un último término que coincide con cero o con el punto en que se unen las rectas convergentes ${ }^{24}$.

Torricelli encontraba así un límite para las sucesiones infinitas, logrando que su suma fuera determinada. Veremos después a Hobbes enfrentándose a Wallis a causa de esta misma interpretación ${ }^{25}$. El gran matemático de Faenza superaba en esto a Hipócrates por cuanto no necesitaba identificar los bordes rectilíneos de los últimos triángulos con la línea parabólica, y también a Arquímedes, puesto que se atrevió a desafiar al infinito.

21 Elementos X, teorema 1: “Dadas dos magnitudes desiguales, si se quita de la mayor una [magnitud] mayor que su mitad, y de la que queda, una magnitud mayor que su mitad y así sucesivamente, quedará una magnitud que será menor que la magnitud menor dada.» Según cuenta Arquímedes [en "Carta a Eratóstenes" ( $E l$ Método), "Carta a Dositeo" (Prefacio Sobre la esfera y el cilindro I) y en el Prefacio de La cuadratura de la parábola], Eudoxo fue el primero en utilizarlo de un modo expreso; Cf. Vega (1990).

22 Uno de los procedimientos empleados por Arquímedes para hallar la cuadratura de la parábola consiste en aproximar a esta sucesivos triángulos, cada vez más pequeños; después, demuestra por medio de un razonamiento de doble reducción al absurdo, que el área del segmento no es ni mayor ni menor que la suma de esos triángulos. Cf. Dijksterhuis (1987).

23 Lemas XXVI y XXVII, Opere Scelte, p. 392

24 Aún no sabemos si ese punto posee magnitud.

25 Hobbes rechazó la posibilidad de comparar infinitos y que estos pudieran, en algún momento, tener un límite o fin. Cf. Douglas M. Jesseph, Squaring the Circle: The War between Hobbes and Wallis, Univ. of Chicago Press, 1992, pp. 182-183. 
Apoyado en los enunciados anteriores, Torricelli formulaba el Lema XXVIII: Dadas dos progresiones geométricas decrecientes infinitas, tales que sus respectivos términos son proporcionales uno a uno, se cumple entonces el teorema V.12 de Euclides ${ }^{26}$ para un número infinito de magnitudes.

La incorporación de este lema ofrece ahora la posibilidad de comprender los conceptos omnes de Cavalieri en forma de sumas infinitas, evitando, además, el uso excesivo del argumento inductivo.

Con todo este exordio, Torricelli intentaba explicar que "la parábola es un agregado de infinitas magnitudes en proporción cuádrupla»" ${ }^{27}$, como había probado Arquímedes aunque sin mencionar cantidades infinitas; estas magnitudes son pequeños triángulos, cada uno de los cuales constituye un indivisible, y juntos, conforman una serie cuyo límite (decimos nosotros) nos proporciona la medida del segmento parabólico. Estos indivisibles poseen una magnitud finita que disminuye progresivamente hasta anularse.

El infinito que hemos manejado en esta ocasión es el denominado potenciah el que postula que siempre podemos añadir algo más a una cantidad dada: el infinito inalcanzable de los geómetras y el único concebible fuera del ámbito de la teología. En cambio, las colecciones indeterminadas de indivisibles se interpretaron siempre como actualizaciones de ese infinito, lo cual suscitaba una sensación de inquietud y malestar en los científicos de la época ${ }^{28}$; así vemos a Torricelli aludir a cantidades infinitas de indivisibles sólo cuando maneja progresiones decrecientes, y en ese caso, el infinito insinuado es el convencional.

John Wallis advirtió enseguida las ventajas que ofrecían las construcciones basadas en el método de indivisibles e intentó vencer esos escollos que perju-

26 «Si de cualquier número de magnitudes, cada una de las antecedentes tiene la misma razón con cada una de las consecuentes, en la misma razón estarán todos los antecedentes y todos los consecuentes."

Opere Scelte, pp. 394-395

28 Fueron muy numerosas y diversas las objeciones formuladas contra el método de los indivisibles; ver la bibliografia mencionada y E. Festa, "Quelques aspects de la controverse sur les indivisibles", Geometria e atomismo nella Scuola Galileiana, Biblioteca di Nuncius, Studi e Testi, Firenze, 1992, donde el autor comenta cómo el Colegio Romano temió que este método escondiera un atomismo de corte materialista. Aquí nos interesa subrayar el posible uso de colecciones infinitas y su derivación hacia los desarrollos en serie; en este sentido, cobra especial interés la creación de los infinitésimos. 
dicaban su asentamiento definitivo. Por este motivo, decidió usar sumas infinitas o series de elementos de magnitud infinitesimal, y descubrió dos técnicas para hallar las superficies y los volúmenes de figuras curvas:

- usando indivisibles de la misma medida infinitesimal;

- mediante figuras de magnitud progresivamente inferior.

\section{Los infinitos de John Wallis}

En 1656, Wallis publicó su Arithmetica infinitorum ${ }^{29}$. En el Prefacio cuenta cómo se había familiarizado con el método de indivisibles gracias a la Opera Geometrica de Torricelli. Esto nos induce a pensar que no fue Cavalieri su fuente de inspiración; lo cual, además, parece haber sido un golpe de suerte y no un demérito para el Profesor Saviliano: «Si Wallis hubiera tenido a su alcance la Geometría de Cavalieri, sin duda se hubiera sentido repelido por lo prolijo y complicado de esta obra, ${ }^{30}$. La influencia directa de Torricelli indica un uso diferente de esos indivisibles: la posibilidad de tratar las magnitudes omnes como sumas de elementos homogéneos. Wallis también se aventuró a dar ese salto que precisaba la Geometría: introducir el álgebra en sus deducciones ${ }^{31}$.

Los indivisibles de Wallis poseen cantidad, como los de Torricelli: «Las líneas tienen que ser comprendidas con anchura, y lo mismo los planos" ${ }^{32}$. Y sus agregados o sumas se identificarán con las figuras respectivas.

La técnica de Wallis consiste en rellenar una superficie (o un sólido) con paralelogramos (paralelepípedos) de anchura infinitesimal y uniforme, siempre la misma, en una cantidad infinita. En la figura anterior observamos un triángulo en cuyo interior hemos colocado rectángulos de idéntica anchura, pero de

29 En Opera Mathematica, 1693-1699, vol. I. Ver J.F. Scott, The Mathematical Work of John Wallis, Chelsea Publishing Co., New York, 1981.

30 Aproximadamente este es el comentario de De Gandt (1987) op. cit., p.152.

31 Wallis concedió primacía al álgebra sobre la geometría e intentó fundamentar los argumentos geométricos en deducciones algebraicas. Ver su Mathesis Universalis (1657), en Opera Matemática I.

32 Treatise of Algebra, printed by John Playford, for Richard Davis, Univ. of Oxford, 1685, p. 285. 


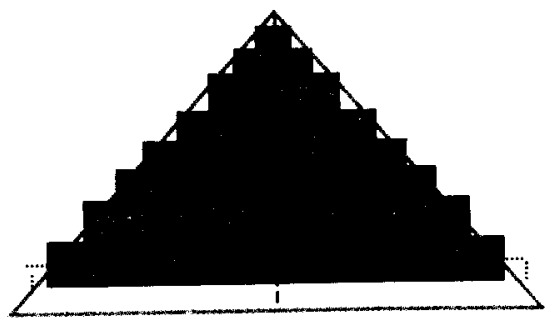

Figura 2.

longitud variable, de tal modo que al disminuir aquella, conseguimos aproximar los paralelogramos a los lados del triángulo, obteniendo un error o diferencia progresivamente menor.

Esa anchura ha de ser la mínima posible: un infinitésimo de la altura del triángulo. Por primera vez en la historia de la Matemática hallamos infinitésimos, estos son una invención de Wallis ${ }^{33}$. Surgen del cociente entre una magnitud finita y otra infinita, como si ese infinito pudiera ser actualizado y nos descubriera, de pronto, infinitas partes en una magnitud concreta. Pero este procedimiento no es más que una conjetura, pues concebir un infinito absoluto actualizado no pasa de ser una suposición útil ${ }^{34}$.

El infinito absoluto de Wallis ${ }^{35}$ es una cantidad mayor que cualquiera [toda] otra asignable, por consiguiente, el infinitésimo, expresado al modo de los racionales, $\delta, 1 / \infty$, traduce el hecho de partir una superficie o volumen en infinitas porciones pequeñísimas. Así, cada uno de los rectángulos del diagrama anterior tiene una anchura igual a $1 / \infty \mathrm{de} h$. Su argumento resuelve la paradoja formulada por Zenón ${ }^{36}$ : la adición de infinitas partes finitas iguales determina necesariamente una magnitud infinita; aquí, en cambio,

33 Leer a M. Sellés acerca de la diferencia entre los conceptos de indivisibles e infinitesimales y la interpretación de Wallis al respecto, en "Isaac Newton y el infinitesimal», Theoria (1999), vol. 14 (3), 2. época, pp. 431-460; aqui, pp. $441-444$.

34 Tal es la opinión de Wallis en Philosophical Transactions, Opera Mathematica, II, p. 2242, según indica A. Malet, From Indivisibles to Infinitesimals, Enrahonar, Barcelona, 1996, p. 40 y Jesseph (1992), p. 185. También en "Carta a V. Léotaud", incluida en Defense of the Angle of Contact, O.P. I, p. 80.

35 M. Sellés opina que se trata de un infinito potencial actualizado. Conversaciones.

36 E. Rufini, IlMetodo di Archimede e le orogini del calcolo infinitesimal nell'Antichita,Feltrinelli,Milano, 1961. 
la suma de infinitas partes infinitésimas nos proporciona una magnitud finita ${ }^{37}$ :

$$
\begin{gathered}
\frac{1}{\infty} \cdot \infty=1 \\
\left(\frac{1}{\infty} \cdot h\right) \cdot \infty=h
\end{gathered}
$$

La genialidad de Wallis desvanece el viejo impedimento acerca del modo de componer razones y proporciones, pues cada uno de esos indivisibles es homogéneo a la magnitud total, aunque su medida sea inferior a toda magnitud finita.

Las líneas de Wallis son dilatables ${ }^{38}$, se ensanchan al ser multiplicadas. Y lo mismo le ocurrirá a los planos de espesor infinitesimal que componen un sólido. Con esta idea evita saltos o fisuras en el continuo.

Las magnitudes finitas admiten de igual forma una partición infinita, siempre que las partes se ordenen en progresión decreciente, tal como vimos en la cuadratura de la parábola de Torricelli. Se presenta, entonces, a Wallis esa doble posibilidad de utilizar sus infinitos:

1) De esta nueva manera, con partes alícuotas ${ }^{39}$ de la misma medida infinitésima y en número infinito: $(1 / \infty+1 / \infty+1 / \infty+\ldots) \cdot h=b$

2) Al modo del sabio de Faenza: una cantidad ilimitada de partes cada vez más pequeñas, pero finitas, hasta completar la figura deseada: $(1 / 2+1 / 4+$ $1 / 8+\ldots) \cdot h=h$

$Y$ de estas dos formas resolverá las cuadraturas e intentará construir sólidos de superficie ilimitada y volumen fijo. Aunque debemos subrayar que ambos infinitos son muy distintos, porque ¿cómo una cantidad infinita de infinitési-

37 En Arithmetica Infinitorum, O.P. I, p. 454: "Cum enim infinite parvum infinites multiplicatur, assurgit non nunquam quantitas fortis magna, nempe illa ipsa cujus illa fuit aliquota pars utut infinite parva. Nam $1 / \infty . \infty=1 ; 1 / \infty \mathrm{A} \cdot \infty=\mathrm{A}$.) Ver A. Malet, concretamente, p. 48.

38 Arithmetica Infinitorum, O.P. I, p. 297: "... in hoc saltem differunt, quod linea supponitur dilatabilis esse, sive tantillam saltem spissitudinem habere ut infinita multiplicatione certam tandem altitudinem sive latitudinem possit acquirere, tantam nempe quanta est figurae altitudo." J. F. Scott (op. cit., p. 19) afirma que «esto es nada menos que la noción de la generación de cantidades, base del Método de Fluxiones."

39 Alícuotas respecto al espesor o altura; no en cuanto a su longitud. 
mos es igual a un número infinito de partes finitas? La solución estriba en la posibilidad lógica de actualizar o no ese infinito tradicional.

Hay razón entre lo infinito y lo finito, aunque esta razón no es finita:

"Lo infinito es a lo finito como lo finito es a lo infinitamente pequeño, y esta es una razón infinitamente grande. Análogamente, lo finito es a lo infinito como lo infinitamente exiguo es a lo finito, y ahora esta es una relación infinitamente pequeña" ${ }^{40}$

Nada se puede decir en contra de las magnitudes infinitésimas de Wallis: su extensión es tan pequeña que se introducen por los resquicios que dejaba libres el método de exhausción. Su medida es prácticamente nula, o non-quanta ${ }^{41}$, pero creemos que estas palabras significan ausencia de cantidad finita ${ }^{42}$, o aquello que no puede ser percibido de una forma natural sino aplicando algún tipo de instrumento o técnica ${ }^{43}$. Las líneas y los planos de espesor infinitesimal poseen una dimensión más que la que les concediera la geometría de los Elementos, y por ello, su agregado o conjunto se interpreta como la suma de esas líneas y de esos planos.

Hemos hablado de ese infinito que consigue hacer a las magnitudes infini-

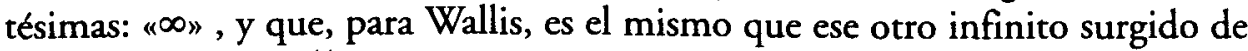
la expresión: «1/0" 44 . Lo descubre a partir de la sucesión de los números naturales comenzando por cero y de sus respectivos recíprocos:

$$
0,1,2, \ldots, \quad ; \quad \frac{1}{0}, \frac{1}{1}, \frac{1}{2}, \ldots
$$

40 Carta a Léotaud, Defense, p. 80: "Concedo Infiniti ad Finitum, nullam esse Finitam Rationem ... Adeoque, Infiniti ad Finitum, aut etiam Finiti ad Infinite-exiguum, rationem esse dico Infinite-Magnam; Finite ad Infinitum, vel etiam Infinite-exigui ad Finitum, rationem Infinite-exiguam."

41 Arithmetica Infinitorum (p. 297): “Nam parallelogrammun cujus altitudo supponitur infinite parva, hoc est, nulla, (nam quantitas infinite parva perinde est atque non-quanta)."

42 Malet (1996), pp. 35-48.

43 Interpretación de A. Robinson, Non-Standard Analysis, North Holland Publishing Co., Amsterdam, 1974. Ver también R. Kossak, "What are Infinitesimals and why They cannot be seen?", American Mathematical Monthly (1996) vol. 103 (10), pp. 846-853.

44 "Cum enim primus terminus in Serie Primanorum sit 0; primus terminus in serie reciproca erit $\infty$ vel infinitus (sicut in divisione, si diviso sit 0 , quotiens erit infinitus.) Cita tomada de Scott, op. cit, p. 20. 


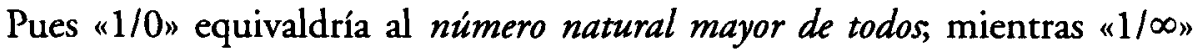
sería el menor número: el cero. Al considerar este infinito como absoluto, obtenemos esos infinitésimos que parecen ser nada. Wallis quedaba atrapado entre sus propias palabras y Thomas Hobbes supo sacar buen partido de ello en sus Six Lessons to the Professors of the Mathematiques (1656): tomando como referencia la Geometría de Cavalieri, denunció a Wallis el significado, en su opinión, contradictorio e imposible de sus infinitésimos; aunque, bien es verdad, él mismo había realizado una lectura completamente equivocada de la obra del jesuato, descubriendo indivisibles extensos donde no los había ${ }^{45}$. Hobbes nunca comprendió el alcance de los infinitésimos de Wallis.

\section{Las series algebraicas}

La Géométrie (1637) de Descartes difundida por Van Schooten y los contactos con Roberval y Fermat, resultaron decisivos en el análisis de Wallis. Sus desarrollos algebraicos incidieron en la geometría, desvinculándose de lo que siempre había sido un lenguaje encorsetado con fórmulas de proporcionalidad entre magnitudes. Omnes lineae, a partir de cero, son denominadas por Wallis series aritméticas; manejadas ahora como sumas de sucesiones tendentes a un límite ${ }^{46}$. Al diseñar cuadraturas, indaga la razón existente entre las líneas de la figura cuestionada y las respectivas de un paralelogramo circunscrito a la misma. Practica aplicando su método inductivo ${ }^{47}$ con secuencias de números naturales, desde cero hasta cierta cantidad, $m$, que coincide con la medida de la base del paralelogramo. Asigna una medida a cada línea, aunque prescinde de su grosor, suponiéndolo uniforme e infinitesimal.

45 Cf. Jesseph (1992), p. 181 y ss.

46 E. Giusti (1980, p. 39) comenta que «tan pronto como la teoría geométrica de magnitudes fue abandonada y sustituida por técnicas algebraicas, la atribución de una altura infinitesimal a las líneas de una figura, representará el ajuste necesario para obtener un resultado finito desde una suma infinita, y será posible entonces hablar explícitamente de suma de todas las lineas."

47 Método de inducción incompleta, criticado por Hobbes (cf. D. M. Jesseph, 1992, pp.173 y ss.) y Fermat; este último afirmó que: «su modo de demostrar, fundado en la inducción más que en un razonamiento al estilo de Arquímedes, perjudicará a los novatos, quienes necesitan silogismos demostrativos, desde el principio hasta el fin ." (cita de M. Baron, The Origins of the Infinitesimal Calculus, Dover, New York, 1987, p.212. 
Su explicación comienza en lo más simple: intenta estudiar la superficie de un triángulo; para ello, lo encierra en un rectángulo de la misma base y altura y traza todas las líneas paralelas a la base desde el vértice, componiendo entonces la sucesión: $0,1,2, \ldots m$. El rectángulo estará formado por las líneas: $m+$ $m+\ldots+m$, en una cantidad igual a $(m+1)$.

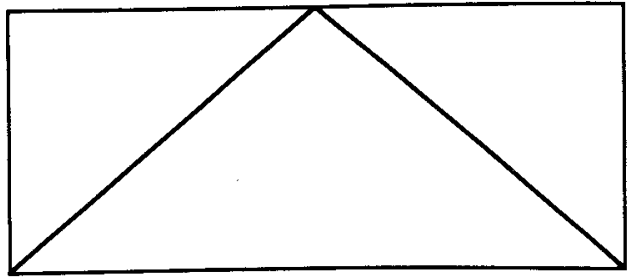

Figura 3.

La razón entre las superficies de ambas figuras se expresa así:

$$
\frac{0+1+2+\ldots+m}{m+m+m+\ldots+m}
$$

Y su valor ya lo conocemos, es $1 / 2$, porque es ésta la razón entre el triángulo y el rectángulo que lo circunscribe. Luego, prueba con el agregado de los círculos que forman un cono, relacionándolos con los respectivos del cilindro que lo circunscribe; suponiendo que los diámetros de los círculos del cono se incrementen aritméticamente, los respectivos del cilindro lo harán según los cuadrados de su diámetro. Así, la razón entre ambas figuras es:

$$
\frac{0^{2}+1^{2}+2^{2}+\ldots+m^{2}}{m^{2}+m^{2}+m^{2}+\ldots+m^{2}}
$$

La cual es 1/3, porque esta es la medida expresada por Arquímedes. Tras examinar otras figuras y otras series, determina la forma general que ha de tener este tipo de relaciones:

$$
\frac{0^{n}+1^{n}+2^{n}+\ldots+m^{n}}{m^{n}+m^{n}+m^{n}+\ldots+m^{n}}
$$

Wallis comprueba que la expresión anterior logra igualarse a $1 / n+1$ más cierta cantidad que disminuye con relación al número de líneas que intervienen en el cálculo; cuando la serie es infinita, ese residuo desaparece por com- 
pleto. Hablamos ya del limite de una serie convergente o cantidad a la que esta se aproxima y que Cauchy lo identificará con su suma. Pero, como ha señalado Jesseph, en este razonamiento se intenta asignar un valor finito a una suma infinita sin el respaldo de ningún tipo de axioma o principio que lo garantice ${ }^{48}$.

El resultado es semejante al que obtuvieron Arquímedes y Cavalieri, aunque ellos no advirtieron esa pequeña diferencia que únicamente se descubre gracias a la formalización algebraica ${ }^{49}$ :

$$
\begin{aligned}
& \cdot \frac{0+1}{1+1}=\frac{1}{3}+\frac{1}{6 \cdot 1} \\
& \cdot \frac{0+1+4}{4+4+4}=\frac{1}{3}+\frac{1}{6 \cdot 2} \\
& \cdot \frac{0+1+4+9}{9+9+9+9}=\frac{1}{3}+\frac{1}{6 \cdot 3} \\
& -\ldots \ldots \ldots \ldots \ldots \ldots \\
& \cdot \frac{0+1+4+9+\ldots}{m+m+m+\ldots}=\frac{1}{3}+\frac{1}{6 \cdot(m-1)}
\end{aligned}
$$

Esto mismo se puede comprobar para series de otras potencias, incluso con exponentes (indices) racionales e irracionales. Wallis obtuvo así sus resultados, practicando sin descanso con toda clase de ${ }^{50}$ números. Compone y divide estas series observando que el producto de dos cualesquiera de ellas nos proporciona otra serie cuyo respectivo índice es la suma de los índices anteriores; al dividir, el nuevo índice resulta de la diferencia de los dos primeros. No obstante, cuando intenta hallar cuadraturas de funciones hiperbólicas, encuentra cantidades negativas y con ellas, nuevos e insospechados problemas. La relación inversa entre abscisas y ordenadas, característica de este tipo de curvas, modifica la razón expuesta en su fórmula: ahora, el para-

48 Jesseph (1992), p. 177.

49 Ver Scott, op. cit, cap. IV. También I. Grattan-Guinness (ed.), From the Calculus to set Theory 1630-1910, Princeton University Press, 1992, pp. 37-42.

so Baron comenta al respecto: «Lo que constituyó un avance novedoso y apasionante en la Arithmetica fue precisamente el extender esos resultados a exponentes fraccionarios, cero y negativos; y la notación empleada». 
lelogramo de referencia está inscrito en la curva. Este resultado inquietó a Wallis, el cual buscó el modo de envolverlo en una explicación coherente. Nos enfrentamos a tres posibilidades:

1. Que la ecuación de la curva sea del tipo $y=1 / x$; cuyo índice es $(-1)$. Sustituyendo en la fórmula de Wallis, tenemos la razón que expresa su cuadratura :

$$
\frac{1}{-1+1}=\frac{1}{0}=\infty
$$

De esta consecuencia inferimos que la superficie barrida por la curva es ilimitada; lo cual es bastante razonable, dadas sus propiedades.

2. Si la ecuacióni es $y=1 / \sqrt{ } x=1 / x^{1 / 2}$, el índice de la serie respectiva es $(1 / 2)$, por tanto, la razón buscada es

$$
\frac{1}{(-1 / 2)}=\frac{1}{1 / 2}=2
$$

Esto significa que la medida del área encerrada por la curva es el doble que la del rectángulo inscrito en su interior. Este resultado le da que pensar, pues en principio, la superficie limitada por una de las ramas de la hipérbola debería ser infinita.

3. La ecuación es $y=1 / x^{2}$; el índice en este caso es (-2), y la razón que nos interesa es

$$
\frac{1}{(-2+1)}=\frac{1}{1 / 2}=\text { ?? }
$$

Este último resultado sorprendió desagradablemente a Wallis y trató por todos los medios de descubrir en él algún significado aceptable. Por ello, examinó cuidadosamente cada una de las razones y sus índices respectivos; luego, las dispuso ordenadamente:

- Sucesión de índices: $\ldots 4,3,2,1,0,-1,-2,-3,-4, \ldots$

- Sucesión de razones: $\ldots \frac{1}{5}, \frac{1}{4}, \frac{1}{3}, \frac{1}{2}, 1, \infty,-\frac{1}{2},-\frac{1}{3}, \ldots$

Y concluyó que si las razones aumentaban de izquierda a derecha hasta alcanzar el valor infinito, entonces, continuando ese mismo orden, los núme- 
ros negativos indicaban cantidades mayores que infinito ${ }^{51}$, y en esta situación se hallan todas las curvas que obedecen a la ecuación $y=a / x^{n}$, siendo $n>-1$

5. Búsqueda del sólido de volumen finito y dimensiones infinitas

- En el Scholium de la Arithmetica infinitorum Wallis aludió a este tema y nos proporcionó uno de esos modelos que se han mencionado. Dada la dificultad de su lectura, presentamos sólo un ejemplo que ilustra el camino abordado por su autor ${ }^{52}$.

Sea una serie Quadratis subtertianorum reciproca, la cual expresada algebraicamente corresponde a la ecuación:

$$
y=\frac{1}{\sqrt[3]{x^{2}}}
$$

y cuyo índice, obviamente, es $-2 / 3$. La rotación de la curva alrededor de uno de sus ejes engendra un sólido que podemos rellenar con círculos proporcionales a los cuadrados de sus diámetros respectivos; para ello, únicamente tenemos que hallar el producto de dos series idénticas de índice $-1 / 3$, y sumando esos índices obtenemos el propuesto:- $2 / 3^{53}$. De esta manera, logramos que los círculos dibujen el contorno del hiperboloide. La razón que buscamos del sólido respecto al prisma inscrito es $1 /(-2 / 3)+1=3$. Lo cual significa que el hiperboloide, infinitamente alto, mide tres veces el paralelepípedo situado en su interior, y cuyas dimensiones son perfectamente calculables ${ }^{54}$.

Generalizando este caso, Wallis llama la atención acerca del modo óptimo de conseguir cuerpos que cumplan las propiedades del sólido de Torricelli: Bastará con encontrar curvas de la familia de la hipérbola - porque entonces sus dimensiones son indefinidas- y cuyos índices, $i$, estén comprendidos

51 «Rationem plusquam infinitam; qualem nempe habere supponatur numerus positivus ad numerus negativum, sive minorem nihilo." Cita tomada de Scott, op. cit., p. 45.

52 Arithmetica, pp. 412-413.

$53 \frac{1}{\sqrt[3]{x}} \cdot \frac{1}{\sqrt[3]{x}}=x^{-1 / 3} \cdot x^{-1 / 3}=x^{-2 / 3}$

54 Los planos que lo componen responden a la serie de índice 1/3. 


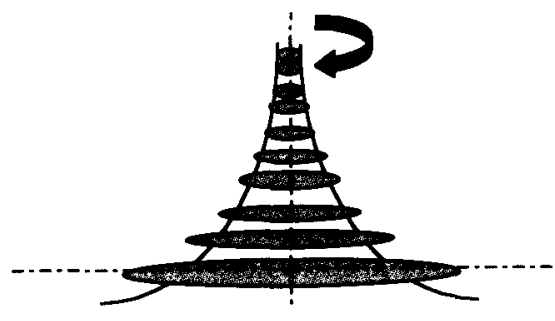

Figura 4.

entre cero y menos uno: $0>i>-1$. Así, las razones halladas son siempre positivas y los volúmenes de los sólidos engendrados por esas curvas, finitos.

El método que propone Wallis es puramente algebraico e inverso al procedimiento que sigue la geometría clásica: No se parte de unas figuras de las que necesitamos conocer sus propiedades, sino que obtenemos esas hipotéticas figuras tras combinar diversas series de números y examinar sus posibilidades. El contexto del descubrimiento es más práctico que deductivo y en el dominio justificativo no hay una fase de formalización: existe un vacío axiomático. El arquetipo euclídeo se inquieta con la brusca irrupción de cantidades expresadas mediante números, ya sean racionales o irracionales, incluso negativas. El infinito también tiene cabida en esta naciente geometría analítica, vinculada aún al uso de la proporcionalidad como medio de hallar las superficies y los volúmenes de las figuras curvas y, por tanto, de medida difícilmente expresable. La condición de homogeneidad defendida en su día por el axioma de Eudoxo carece de importancia, pues el álgebra, como hemos visto tantas veces, opera con cantidades sin preocuparse de la quizá cuestionable homogeneidad de las magnitudes a las que aquellas representan ${ }^{55}$.

- El segundo modelo, lo describió Wallis casi tres décadas después, en el Treatise of Algebra de 1685, y se apoya en el desarrollo de las progresiones geométricas infinitas.

Wallis las define como series cuya suma es muchas veces una cantidad finita y asume su deuda con Arquímedes (en la Cuadratura de la parábola),

55 Mathesis Universalis, O.M. I, p. 36. Ver Jesseph (1992, pp. 142 y ss.). También J. L. Gardies, L'héritage épistémologique d'Éudoxe de Cnide. Vrin Paris, 1988. 
Torricelli (De dimensione parabolae) y Gregorio de San Vicente (Quadratura circuli, 1647) ${ }^{56}$.

De Torricelli tomó los lemas XXIV a XXVII de la obra aludida y que ya hemos expuesto. A partir de ellos, construirá "de un modo más general" ${ }^{57}$ sólidos que, aun teniendo dimensión ilimitada, posean un volumen fijo y determinable.

Wallis aplica su método inductivo al análisis de sucesiones de números decimales, introduciendo inicialmente el lema XXVI de Torricelli, por el cual sabemos que la suma de los términos de una progresión infinita decreciente es finita. Enuncia además las siguientes proposiciones:

1) «Si de una cantidad propuesta tomamos la mitad, y luego la mitad del resto, y así infinitamente, la suma de todo será igual a la cantidad propuesta.»

Wallis comprueba que el valor al que se aproxima la suma de la siguiente sucesión: $0.5,0.25,0.125,0.0625, \ldots$ es 1 .

2) «Si de una cantidad propuesta tomamos menos de la mitad, y luego otra vez, en la misma proporción, menos de la mitad, y así sucesivamente, la suma total será una cantidad menor que la dada.»

3) "Si tomamos ahora una tercera parte de una cantidad dada, luego otra tercera parte, etc., la cantidad que obtenemos al final será la mitad de la inicial."

4) «Si hacemos la misma operación, pero esta vez tomando una cuarta parte, el resultado final será un tercio de la cantidad propuesta.»

Después, toma una quinta parte, luego una sexta, etc. Hasta que en la octava proposición, Wallis formula la expresión que generaliza su investigación:

8) "Si de cierta magnitud dada, $M$, tomamos una parte denominada $a$, y del resto, otra parte denominada $a$, y así infinitamente, el total de la progresión será una parte denominada $a-1 . »$ es decir:

56 Utilizó una técnica de cuadratura denominada "ductus plani in planum», en Opus Geometricum quadrature circuli et sectionum coni. Ver Baron (1987), op. cit., pp. 135 y ss. y Boyer (1985), op. cit., p. 137.

57 Treatise, p. 355. 


$$
\left(\frac{1}{a}+\frac{1}{a a}+\frac{1}{a a a}+\ldots\right) \cdot M=\frac{1}{a-1} \cdot M
$$

Todavía no ha efectuado ninguna deducción matemática, sino aproximaciones decimales. Recupera de su Arithmetica la expresión que le permite generalizar el valor de la suma de los términos de una progresión geométrica finita ${ }^{58}$ :

$$
S=\frac{V R-A}{R-1}
$$

Donde $V$ es el término mayor; $A$, el menor, y $R$, el multiplicador o exponente de la razón. La expresión [2] podemos transformarla en la siguiente:

$$
S=\frac{V R}{R-1}-\frac{A}{R-1}
$$

Pero si la progresión fuera infinita, la parte tomada (1/aaaaaa ...) sería infinitamente pequeña y $A / R$ - Iterminaría desapareciendo; por lo cual, la expresión [3] se transformaría en

$$
\mathrm{S}=\frac{V R}{R-1}
$$

La suma de los términos de una progresión cualquiera puede definirse también enumerando sus elementos:

$$
S=V+\frac{V}{R}+\frac{V}{R R}+\frac{V}{R R R}+\ldots=\frac{V R}{R-1}
$$

Operamos en esta ecuación dividiendo ${ }^{59}$ por $\mathrm{R}$ :

$$
\mathrm{S}^{*} \Rightarrow \frac{V}{R}+\frac{V}{R R}+\frac{V}{R R R}+\ldots=\frac{V R}{R-1}
$$

58 «Si terminus primus seu minimus dicatur $A$, maximus $V$, communis ratio $R$, et progressionis summa $S$, erit . Arithmetica, cap. XXVI. En realidad, esta fórmula se ajusta a las progresiones crecientes; cuando se trata de una progresión decreciente, $V$ es el término mayor y primero; $A$, es el último y más pequeńo; y $R$ es el inverso de la razón. Wallis opera con números racionales. de $S$.

59 Wallis omite dividir el término $S$ entre esta misma $R$, por ello, hemos escrito $S^{*}$ en lugar 
Si suponemos ahora que $V=1$ y $R=a$, obtenemos la siguiente ecuación, idéntica a [1]

$$
S^{*} \Rightarrow \frac{1}{a}+\frac{1}{a a}+\frac{1}{a a a}+\ldots=\frac{1}{a-1}
$$

Es decir, la suma de los términos de una progresión geométrica decreciente está en la relación $1 / a-1$, siendo «a) la parte tomada, es decir, el inverso de la razón ${ }^{60}$. Wallis ha logrado modelar una demostración que sustituya a la inferencia inductiva.

La fórmula [5] permitirá hallar, directamente, la cuadratura de la parábola, tal como la concibieran Arquímedes y más adelante Torricelli. La suma de los triángulos que podemos inscribir en un segmento parabólico se expresa así $^{61}$ :

$$
\Sigma+2(1 / 8 \Sigma)+4\left(1 / 8^{2} \Sigma\right)+\ldots+2^{n}\left(1 / 8^{n} \Sigma\right)
$$

Por consiguiente,

$$
S=\Sigma+1 / 4 \Sigma+1 / 4^{2} \Sigma+\ldots+1 / 4^{\mathrm{n}} \Sigma
$$

El número de términos de la progresión sería infinito, según Wallis, y su suma finita. Luego aplicando [4]:

$$
S=\Sigma+\frac{1}{3} \Sigma=\frac{4}{3} \Sigma
$$

Wallis no sólo toma partes enteras, también fraccionarias, mayores y menores que la unidad y siempre en una cantidad infinita. Pero antes de tomar todas las partes, si quedara un resto, este sería menor que cualquier magnitud asignada $o$ se desvaneceria. ${ }^{62}$

Los infinitésimos de Wallis se postulan para la geometría y el álgebra del mismo modo en que se introduce ese infinito que los actualiza: haciéndolos homogéneos a las magnitudes de orden inmediatamente superior, con las que guardan una relación infinitamente grande. Sin embargo, para evitar cualquier tipo de objeción, decidió que la magnitud de esos infinitésimos fuera nula.

60 Si tomamos partes $m / n$, debemos poner en la fórmula su inverso: $n / m$.

61 Cf. Dijksterhuis, Archimedes, Princeton Univ. Press, Princeton, New Jersey, 1987

62 Algo así formuló Torricelli en el Escolio al lema XXVII en La cuadratura de la parábola. 
En este punto, el razonamiento de Wallis nos sitúa ante un profundo abismo: ¿son los infinitésimos extensos o no? En un medio estático como es el de la geometría euclídea, no hay posibilidad de escapar'al error y a la contradicción: se es o no se es. Sin embargo, si concebimos los objetos geométricos en movimiento, dilatándose o contrayéndose, sucediéndose con relación al tiempo, abarcando espacios sensibles, entonces, lo que en un principio nos pareció insalvable, ahora lo estimamos plausible. De nuevo, el paradigma euclídeo se alarma, siendo la mecánica la que apremia e incita con mayor fuerza ${ }^{63}$.

Volviendo a la expresión [5], observamos que a puede adquirir diversos valores: ser mayor, menor o igual que la unidad. Dependiendo de esto, el significado de la razón variará.

A. Razón de minoria: a >1.

a. Si la parte tomada, de $M$, es menor que la mitad, el resultado es entonces positivo, pero menor que la cantidad propuesta. Por ejemplo, si tomamos partes iguales a $1 / 4$, entonces

$$
\mathrm{S}=\frac{1}{4-1} \cdot M=\frac{1}{3} \cdot M
$$

b. Si tomamos una parte mayor que la mitad (p.ej., 3/4), la solución será mayor que la cantidad propuesta.

$$
\mathrm{S}=\frac{1}{4 / 3-1} \cdot M=\frac{1}{1 / 3} \cdot M=3 M
$$

c. Si la parte sustraída es igual a la mitad ${ }^{64}$, obtendremos una magnitud equivalente a la inicial:

$$
S=\frac{1}{2-1} \cdot M=M
$$

B. Razón de mayoría: $a<1$. Cuando tomamos partes mayores que la magnitud dada, por ejemplo, 7/6, la magnitud obtenida es más que infinita:

63 Ver Sellés (1999) e "Impacto instantáneo y acción continua en la mecánica de Newton", Éndoxa, Series Filosóficas, n..$^{\circ} 11$ (1998), pp. 9-80. También Malet (1996)

64 Bradwardine trató estas series: $1 / 2+1 / 4+1 / 8+\ldots=1$ 


$$
S=\frac{1}{6 / 7-1} M=\frac{1}{-1 / 7} \cdot M=-7 M=>\infty
$$

C. Razón de igualdad: $a=1$. Las partes sustraídas son iguales a la magnitud; al final obtenemos una cantidad infinita:

$$
\mathrm{S}=\frac{1}{1-1} \cdot M=\frac{1}{0} \cdot M=\infty
$$

Wallis también contempla la posibilidad de obtener partes irracionales o sordas ${ }^{65}$, en cuyo caso se procede operando algebraicamente con exponentes fraccionarios. De todo esto colegimos que la suma de los términos de una progresión infinita alcanza límites insospechados, en función del tamaño de esos mismos términos. Si evitamos que la razón de esa progresión sea mayor o igual que la unidad, la suma de la serie ha de ser finita y conmensurable.

Pero aunque Wallis hable aquí de razones, sus composiciones algebraicas no se fundamentan en las razones existentes entre las superficies curvas y las de los paralelogramos que las circunscriben, como vimos hacer en el apartado anterior, sino que desligándose de la geometría clásica, construye únicamente cadenas algebraicas para determinar esos volúmenes y esas superficies curvas. También Fermat, en su De aequationum (1658), propuso medir la superficie del interior de un segmento hiperbólico mediante la suma de una cantidad indeterminada de paralelogramos y a este método lo denominó logaritmico. Asimismo, Pascal y Roberval construyeron nuevos modelos de cuadraturas ${ }^{66}$.

Al igual que hizo en la Arithmetica, en el Treatise of Algebra Wallis combina dos o más progresiones. Su objetivo consiste en componer superficies y sólidos con los que calcular las áreas y los volúmenes de diversas figuras. Por ejemplo, dadas dos magnitudes, $A$ y $B$, si tomamos en ellas, respectivamente, partes $a$ y $b$, infinitamente, el producto de ambas series ha de ser:

$$
\left(\frac{1}{a} \cdot \frac{1}{b}+\frac{1}{a a} \cdot \frac{1}{b b}+\frac{1}{a a a} \cdot \frac{1}{b b b}+\ldots\right) \cdot A B=\frac{1}{(a b-1)} A B
$$

66 Ver Grattan-Guinness (1992), pp. 42-46. 


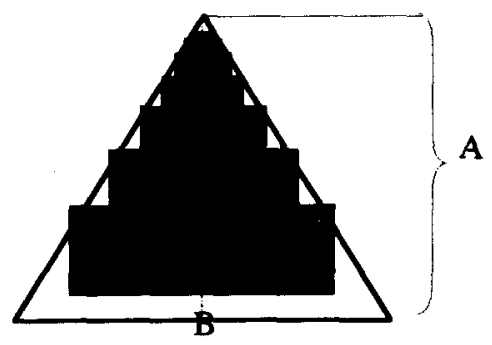

Figura 5.

Mediante este procedimiento, hemos construido planos cuyas dimensiones se hallan sujetas a una ley de crecimiento o disminución controlada. El agregado de los mismos, o su suma, nos proporciona el área de una figura, por ejemplo, la de un triángulo.

Cada uno de los rectángulos tiene una longitud y altura diferentes a su inmediato superior; sin embargo, no son de espesor infinitesimal, pues poseen una medida finita; al disminuir progresivamente según las razones indicadas, $a b$, sus magnitudes tienden a desvanecerse, y en el límite, a desaparecer.

Para medir la superficie del triángulo, precisamos que el producto $a b$ sea igual a 3, lo cual conseguimos si las partes tomadas son, respectivamente, 1/2 y $2 / 3$ de las medidas que van surgiendo.

También podemos encontrar razones de minoría, igualdad y mayoría, pero debemos tener en cuenta ambas partes, $a$ y $b$. Tomamos de nuevo la expresión [6] y observamos que debemos analizar el binomio "ab-1" para determinar cuándo la suma de los paralelogramos es finita, infinita o más que infinita. Nos interesará descartar los sucesos en los que el producto ab $£ 1$. El procedimiento es el mismo que el anterior, aunque conviene analizar cada una de las partes por separado:

a. Sea una de las partes de mayoría y otra de minoría y que cumplan la desigualdad $a b>1$. Entonces, esto significa que los paralelogramos incrementan uno de sus lados pero disminuyen el otro.

b. Si ambas son de minoría, disminuirán progresivamente en ambas direcciones.

c. Cuando una de las partes es de minoría y la otra de igualdad, solamente se reducirá uno de los lados; el otro permanecerá invariable. 
Volviendo al sólido de Torricelli, Wallis advirtió enseguida cómo debía manejar su algoritmo para encontrar el resultado deseado. Primero, necesitaba demostrar que podemos rellenar el interior de una curva dibujada en el plano con líneas ordenadas en progresión decreciente: así, obtendría una superficie finita. Prescindirá del espesor de esas líneas; al menos, no mencionará nada al respecto; por lo cual las concebimos de longitud variable pero idénticas en espesor.

Más adelante, esa curva giraría engendrando un sólido hiperbólico. Wallis deberá entonces componer su interior con paralelepípedos dispuestos también en orden decreciente. Comprobada su viabilidad, obtendrá entonces un cuerpo de volumen determinado y longitud infinita.

Inicia su explicación tomando una hipérbola equilátera, $S H$, cuyos brazos se prolongan indefinidamente; las asíntotas, $A B$ y $A F$, perpendiculares entre sí, se cortan en el punto $A$. Suponemos una magnitud propuesta, $m$, la cual representamos como un segmento recto igual a $D H$ y paralelo a $A F$. Sea ahora $B S$ una parte de $m(1 / a)$ a la que llamamos $d$ decimos entonces que $B S=d m^{67}$

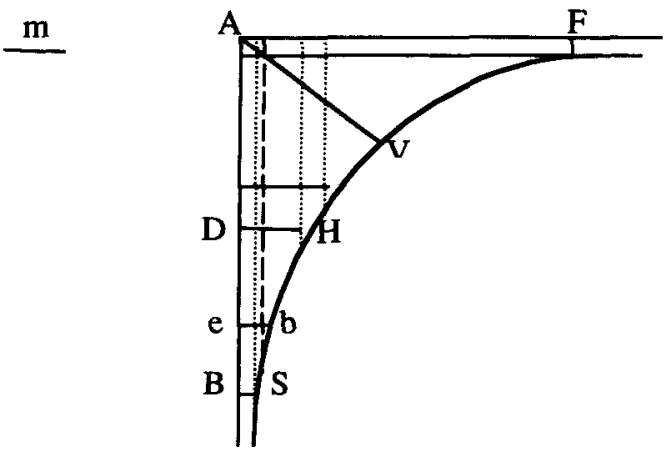

Figura 6 (de Wallis).

67 Treatise, p. 350: "Now suppose we the propose Magnitude $m=D H$ (or, if the Magnitude proposed be not Streight Line, let it at least be represented by such) this I inscribe, Parallel to $A F$, And (the assigned part thereof first to be taken) $m / a=d m=B S$; wich I likewise inscribe parallel to $A F$, (below $D H$; that is, further from $A F$; because by construction, it es lefs than $D H$, as being a part thereof.)". En el dibujo de Wallis las líneas $B S$ y $D H$ se hallan intercambiadas y el segmento eb está por encima de $B S$, lo cual dificulta la deducción, pues la expresión [7] no se cumpliría: así, hemos optado por colocarlas de modo inverso. Sea $B S=1 / a D H$ $=1 / a \cdot m=d m$. 
Wallis prosigue: Tomamos en $B A$ una parte $B e=\delta$, para que siendo $B A=1$ se cumpla la siguiente proporción:

$$
B e: B A:: B S: D H
$$

Es decir, tomamos en $B A$ un segmento, $B e$, que esté respecto a $B A$ en la misma proporción que lo están $B S$ y $D H$. O lo que es lo mismo, como $d m$ está a $m$.

$$
\begin{gathered}
\delta: 1:: B S: m \\
\delta: 1:: d m: m \\
\text { Luego } \delta=d
\end{gathered}
$$

Trazamos ahora eb paralelo a $B S$; sabemos que en las hipérbolas equiláteras los rectángulos inscritos poseen una superficie equivalente pero sus lados son recíprocos; luego como $A e=1-\delta$; entonces afirmamos que

$$
\begin{gathered}
A e: A B:: B S: e b \\
(1-\delta): 1:: d m: e b \\
e b=\frac{d m}{1-d}
\end{gathered}
$$

A continuación, Wallis propone la siguiente igualdad:

$d m=(1-d) \cdot(d m+d d m+d d d m+\ldots)=d m-d d m+d d m-d d d m+d d d m-\ldots$

Es decir, plantea un modo de expresar algebraicamente el producto $d m$. Operando en [8], obtenemos:

$$
e b=d m+d d m+d d d m+\ldots=m(d+d d+d d d+\ldots)
$$

Entonces,

$$
e b=m\left(\frac{1}{a}+\frac{1}{a a}+\frac{1}{a a a}+\ldots\right)
$$

Luego,

$$
e b=\frac{m}{a-1}
$$

Esto significa que cualquier ordenada de la curva ${ }^{68}$ se podría definir en función de los términos de una progresión decreciente infinita, o como un

68 Wallis también contempla el caso en el que eb está situada por debajo de $B S$, siendo entonces $A e=1+\delta$ y eb igual a la suma de los términos de una progresión decreciente, pero de razón dd ó $1 / a a$. 
conjunto de partes de una magnitud dada; por tanto, toda ordenada de la hipérbola es conmensurable y finita. Ahora bien, necesitaríamos demostrar que el agregado de todas esas ordenadas, y ninguna otra línea más, compone la superficie de la hipérbola y que todas ellas se distribuyen según una progresión decreciente.

Wallis tendría que comprobar ahora que así como la ordenada, $B S$, es una parte ( $1 / a)$ de $D H$, y eb es también otra parte de esa misma magnitud, aunque en distinta proporción, las otras ordenadas pertenecen también a esa progresión. Así, por los lemas de Torricelli, el conjunto o la suma de todas las líneas limitadas por uno de los brazos de la hipérbola sería determinable y la superficie descrita por la curva, finita. Pero no hay nada de esto. Posiblemente, a Wallis le parece que ya ha demostrado lo suficiente y añade:

"No está de más señalar que la suma [de los elementos] de esa progresión está representada completamente por una línea externa a la hipérbola, entre las asíntotas $A B$ y $A F$, 69

Esa línea -creemos- coincide con la tangente, pero ¿tangente en qué punto? Desde la perspectiva del paradigma euclídeo, este discurso se revela más intuitivo que riguroso y a él asoman las tendencias poco formalistas de la época, en las que primaban los resultados sobre el aparato deductivo ${ }^{70}$.

Nos queda estudiar la segunda parte de toda esta construcción geométrica, porque, hasta ahora, sólo hemos actuado en el plano; veamos qué sucede en el espacio. La curva de la que partimos la hacemos rotar hasta conseguir un cuerpo hiperbólico. Este no parece tener límites, ni en su base ni en su cuello.

En la proposición 31, Wallis establece las condiciones precisas para componer un sólido de paralelepípedos de tres dimensiones: $A, B$ y $C$. Hacemos que, por ejemplo, la longitud de esos paralelepípedos disminuya en razón $1 / a$,

69 Treatise, p. 350: "This not amis here to remark, that the Sum of such Infinite Progression is fiftly expressed by a Line in the External Hyperbola, between the asymptotes $A B, A F$."

70 Respecto al modo de trabajar de Wallis, Baron (1987, op. cit., p. 212) comenta: "Ia debilidad, por supuesto, en toda esta obra, reside en que el todo no se funda en ninguna estructura de prueba estandarizada, sino en comprobaciones tomadas más o menos al azar desde cualquier fuente y derivadas de algunos métodos que respaldan los resultados." 
$1 / a a, 1 / a a a, \ldots$ de $A$. Que las anchuras lo hagan según $1 / b, 1 / b b, 1 / b b b, \ldots$ de $B$. Y que las alturas se reduzcan en la proporción $1 / c, 1 / c c, 1 / c c c, \ldots$ de $C$. El producto de estas tres series constituye el agregado de todas las figuras que configuran el sólido hiperbólico:

$\left(\frac{1}{a} \cdot \frac{1}{b} \cdot \frac{1}{c}+\frac{1}{a a} \cdot \frac{1}{b b} \cdot \frac{1}{c c}+\frac{1}{a a a} \cdot \frac{1}{b b b} \cdot \frac{1}{c c c}+\ldots\right) \cdot A B C=\frac{1}{a b c-1} \cdot A B C^{1}$

Conviene examinar qué proporción existe entre 1 y $a b c$, es decir, qué valores han de tomar $a, b$ y $c$, para que la expresión anterior nos proporcione un resultado finito:

A. Hay relación de minoría, si $a b c>1$. La cantidad obtenida es conmensurable.

B. De mayoría, cuando $a b c<1$. La magnitud final es mayor que infinita.

C. De igualdad, si $a b c=1$. El resultado es infinito

En el enunciado 33 Wallis complica aún más estos datos, proponiendo que

- $a-1=e$

- $b-1=f$

- $c-1=g$

Las respectivas progresiones serán ahora:

$$
\frac{1}{a}, \frac{e}{a a}, \frac{e e}{a a a}, \ldots ; \frac{1}{b}, \frac{f}{b b}, \frac{f f}{b b b}, \ldots \frac{1}{c}, \frac{g}{c c}, \frac{g g}{c c c}, \ldots \text { de } A B C^{2}
$$

Y operando, tenemos:

$$
\left(\frac{1}{a b c}+\frac{e f g}{a a b b c c}+\frac{e e f f g g}{a a b b b b c c c}+\ldots\right) \cdot A B C=\frac{1}{a b c-e f g} \cdot A B C^{73}
$$

71 Hemos aplicado la fórmula $\frac{V R}{R-1} \Rightarrow \mathrm{S}=\frac{(1 / a b c) \cdot a b c}{a b c-1}=\frac{1}{a b c-1}$

$72 \mathrm{Si}$ tomamos $1 / a$ de $1, \mathrm{y}$ luego $1 / a$ del resto: $[1-(1 / a)=e / a]$, tendremos $1 / a \cdot$ ela $=$ elaa. El segundo resto es e/a - elaa $=$ ela $(1-1 / a)=e e / a a$, luego, la siguiente parte tomada es $1 / \mathrm{a} \cdot$ eelaa $=$ eelaaa. El tercer resto es (ee/aa) $-($ ee/aaa $)=e e e / a a a, y$ así sucesivamente.

73 Análogamente, aplicamos $\frac{V R}{R-1} \Rightarrow S=\frac{(1 / a b c) \cdot a b c / e f(s)}{(a b c / f f g)-1}=\frac{1}{a b c-e f g}$ 
Esta igualdad representa la descomposición de un sólido en un número indeterminado de paralelepípedos, cuyas dimensiones están sometidas a una cierta secuencia o proporción continua. El agregado de todos ellos es como 1: $(a b c-e f g)$. Indagando qué dimensiones debe tener cada uno, y qué relación guardan entre sí las respectivas partes, conseguiremos que el cuerpo en cuestión posea un volumen finito.

Un poco más adelante ${ }^{74}$, Wallis indica la ventaja de que una de las longitudes de esos paralelepípedos, como la altura, fuera invariable; de este modo, prescindiremos de una de las series, obteniendo $S=1 / a b-f^{75}$. Así, mutatis mutandi, debemos realizar estimaciones acerca de las formas más convenientes de construir esas progresiones, analizando en detalle las expresiones:

$$
\text { " } \frac{1}{a b c-e f g} \sigma \frac{1}{a b-f}, e t c \text {. Sus magnitudes son finitas o infinitas, o más }
$$
que infinitas; de acuerdo a cómo sean abc-efg $o$ ab-f, etc., mayores, iguales o menores que $0{ }^{76}$.

Añadiendo que, gracias a la composición de dos o más de estas progresiones, hemos encontrado

«... algunas de esas figuras que pueden ser de longitud infinita, pero de magnitud finita; una de las cuales es la de Torricelli, a la que llama Solidum Hyperbolicum Acutum, y gran cantidad de ellas son tratadas en mi Arithmetick of Infinites, y en mi Commercium Epistolicum con Mons. Fermat y otros" ${ }^{77}$.

A pesar de sus esfuerzos, Wallis no ha conseguido alcanzar una expresión algebraica que generalice los resultados de Torricelli: su fórmula depende demasiado de tanteos y aproximaciones. Sin embargo, desde una perspectiva puramente algebraica, sus desarrollos en serie ofrecen multitud de sugerencias: incluyen sofisticados razonamientos aritméticos e introducen elementos y equivalencias que, en principio, podrían parecer ajenos al discurso geométrico

Treatise, Proposición 34.

75 Igualmente, Wallis habría escrito

76 Treatise, p. 355.

77 Ibid. 
habitual. Lo más sorprendente es observar cómo Wallis ha conseguido dejar atrás esos viejos métodos que aplicaban la teoría eudoxiana de razones para hallar cubaturas y cuadraturas.

La ausencia de una axiomatización previa y de una ordenación hipotéticodeductiva, el uso excesivo del argumento inductivo al enunciar consecuencias sin una justificación formal en la que apoyarse, el pasar por alto algunos detalles en las demostraciones, el no definir o esclarecer muchos de los conceptos utilizados, no hacen sino revelar las dificultades con las que el nuevo arte analítico se encontraba al irrumpir de lleno en la geometría de la mano de John Wallis ${ }^{78}$.

78 Ver Helena Pycior, Symbols, Imposible Numbers, and Geometric Entaglements, Cambridge University Press, 1997. 